\title{
TITLE:
}

\section{A Closer Look at DNA Nanotechnology}

$\operatorname{AUTHOR}(\mathrm{S}):$

Tabata, Osamu

CITATION:

Tabata, Osamu. A Closer Look at DNA Nanotechnology. IEEE Nanotechnology Magazine 2010, 4(4): 13-17

\section{ISSUE DATE:}

2010-12

URL:

http://hdl.handle.net/2433/134559

\section{RIGHT:}

2010 IEEE. Personal use of this material is permitted. Permission from IEEE must be obtained for all other users, including reprinting/ republishing this material for advertising or promotional purposes, creating new collective works for resale or redistribution to servers or lists, or reuse of any copyrighted components of this work in other works. 


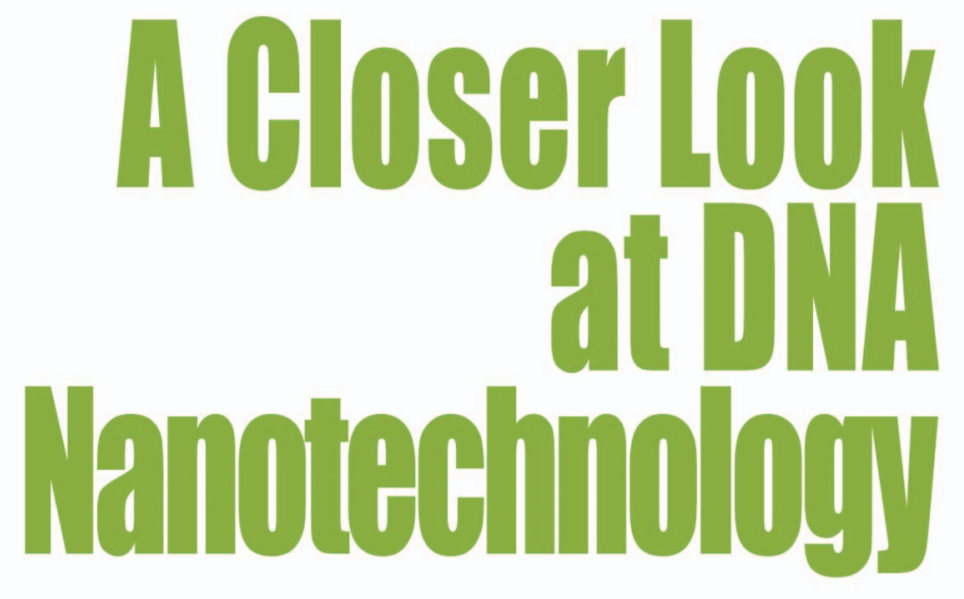

A NANOSYSTEM IN WHICH multiple nanoscale functional components made of a variety of nanomaterials are integrated with a microelectromechanical system (MEMS) becomes increasingly important as a key device for the next generation. A deoxyribonucleic acid (DNA) nanotechnology is expected to play an important role to bridge the gap between the nanoscale components (nanocomponents) and the microscale MEMS as a complementary approach for top-down manufacturing

OSAMU TABATA
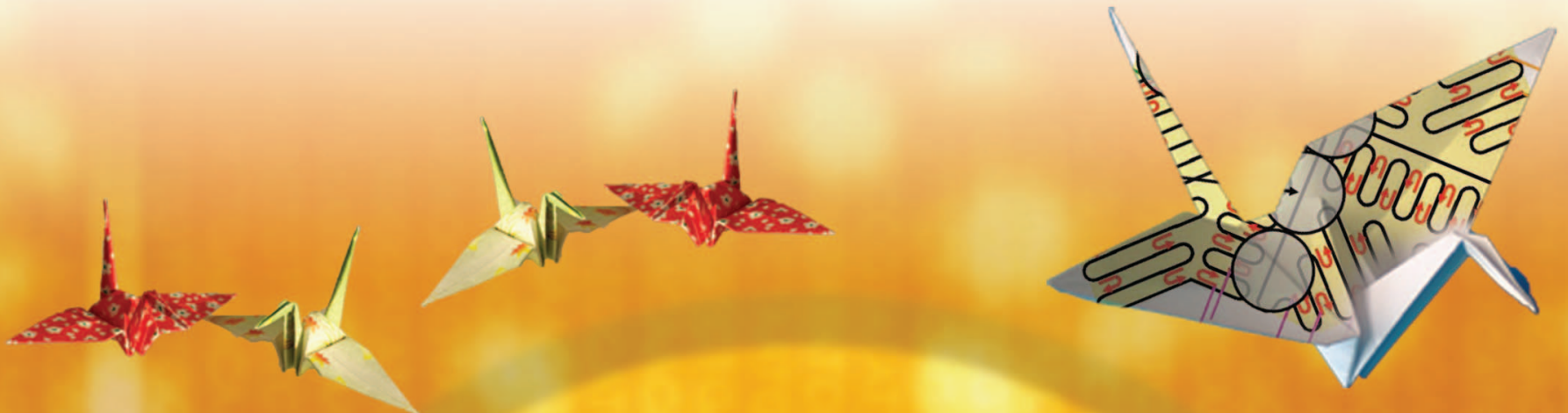

A bridge between nanomaterials and a nanosystem.

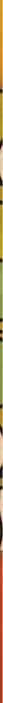




\section{A DNA nanotechnology is expected to play an important role to bridge the gap between the nanoscale components and the microscale MEMS.}

technique and bottom-up manufacturing technique to realize nanosystems. From the design viewpoint of a system, such as large-scale integrated circuits (LSI), MEMS, and nanosystem, it should be noted that the complexity is an essence to generate its functionality. The functionality of LSI/MEMS/nanosystem, where functional components are simply arranged in a periodic order, is limited. Periodic nanostructure can be a part of a nanosystem as a nanocomponent, but it is not enough. Interaction and cooperation of multiple nanocomponents with different features make it possible to generate a unique and useful functionality of a nanosystem.

As nature does, how can we realize a complex nanosystem composed of multiple functional nanocomponents using assembly technique? In case of MEMS and LSI, which is a good example of a system in microscale, a complexity to generate functionality is embedded in a set of photomasks or digital data for electron beam machine to draw patterns. However, so far, no engineering methodology has been realized to assemble multiple functional nanocomponents to specific positions on MEMS/LSI in a specific sequence. This is a challenging goal to address, namely controlling assembly position and sequence of multiple nanoscale functional components made of a variety of nanomaterials to realize a highfunctional nanosystem.

First, a systematic approach termed synthetic engineering for nanosystem (SENS) to categorize assembly technique to realize nanosystem into eightunit operations is introduced. Then, the feature of DNA nanotechnology (pioneered by Seeman in 1982 [2], [3]) and DNA origami technique (proposed by Rothermund in 2006 [4]) are discussed in view of SENS. Then, a concept of a self-organization technique of DNA origami used as a submicroscale functional building block on MEMS according to a given system design is proposed, and an approach using a microfluidic device is

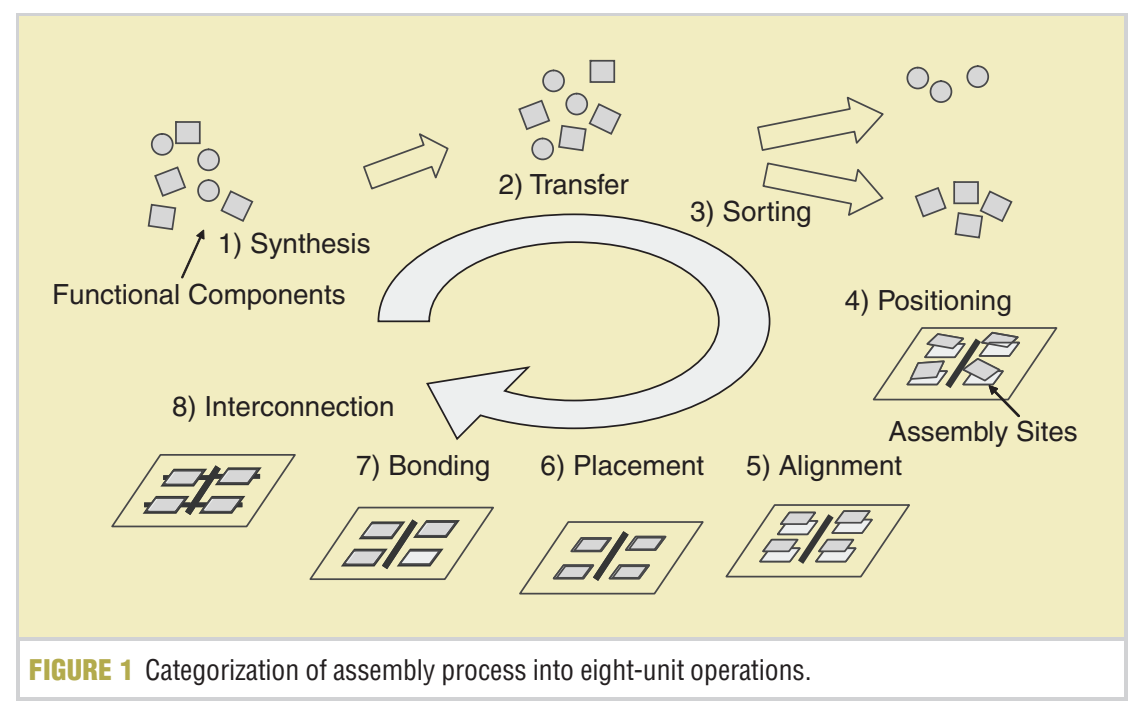

presented. Finally, remaining issues and prospects to be addressed to realize a nanosystem by DNA nanotechnology are overviewed.

\section{SYNTHETIC ENGINEERING FOR NANOSYSTEM}

A sequence of an assembly process can be categorized into eight-unit operations, such as synthesis, transfer, sorting, positioning, alignment, placement, bonding, and interconnection (see Figure 1) [1]. Since an assembly process is considered as a combination of these eight-unit operations in which many process parameters are involved, it is necessary to address an assembly process systematically to optimize it. So, the author termed the systematic approach along this direction as SENS. It should be noted that this categorization is not inherent in micro/nano domain. In any scale, two types of forces are important in an assembly process. One is long-range force to transfer components across the area of interest and to position it; the other is the short-range force acting among the components themselves or component and site on a substrate to place components after alignment. Including an assembly in macrodomain such as an automation system for automotive assembly, any kind of assembly process across the scale can be expressed as the combination of these eight-unit operations. So, SENS can be explained as the establishment of fundamental methodology for automation at nanoscale.

Other than component size, the major differences across the scale are 1) dominant physics to generate force acting on components, 2) magnitude of force acting on components, 3 ) required accuracy of alignment, and 4) measurement resolution for feedback control of force and position. With components size decreasing lower than millimeter scale, an assembly speed lowers because of the difficulty of manipulation (corresponding to transfer, sorting, and positioning) and alignment [5]. To address this drawback, the self-assembly methods have been drawing attention to realize ordered structures by massive parallel production at low cost without external control [6]. Several self-assembly approaches that use interaction force 
between components or components and sites on the substrate based on various physical forces, such as capillary force, hydrophobic force, and electrostatic force, have been proposed. It makes sense to refer these techniques as self-assembly if the target structure has thermodynamic stability. However, according to the definition of Halley and Winkler [7], it is better to call assemble process for a nanosystem in micro- to nanoscale region as self-organization, since self-organization implies a nonequilibrium process. On the other hand, self-assembly is a spontaneous process tending toward equilibrium, namely thermodynamically stable structure. Therefore, SENS should be explained more precisely as a methodology to realize complex arrangement of various functional components that interacts with each other based on a system design by self-organization. However, as described earlier, no methodology has been realized to assemble various functional nanocomponents to specific positions in a specific sequence based on a system design.

\section{DNA NANOTECHNOLOGY}

DNA nanotechnology, proposed by Seeman in 1982, has been growing extremely in the last decade [3],[8]. Especially, DNA origami proposed in 2006 [4] opened up a gate to various researchers to use DNA nanotechnology as their scientific tool. DNA origami uses long scaffold, typically 7,249-nucleotidelong circularsingle-strand Ml3 phage genome and more than 200 short staple strands, to fold the M13 scaffold into an arbitrary twodimensional (2-D) or three-dimensional (3-D) [9] shape with size of a few tens to a few hundreds of nanometer scale. There are four great advantages to use this DNA origami as a nanocomponent.

1) Its surface is precisely addressable.

2) Various nanomaterials, such as nanoparticle [10], nanotube [11], and protein [12], can conjugate with DNA origami. There are several attachment techniques, including utilization of hybridization of single-strand DNA (ssDNA) attached to both nanomaterial and DNA origami.

Controlling assembly position and sequence of multiple nanoscale functional components made of a variety of
nanomaterials to realize a high-functional
nanosystem is a challenging goal.

3) DNA origami can bind each other with the help of hybridization of ssDNA arranged at its edges. So, DNA origami can be used as a building block to construct 2-D or 3-D structure.

4) Origami structure itself can be designed into various $2-\mathrm{D}$ or $3-\mathrm{D}$ shape to increase functionality.

Owing to these advantages, many attempts, including nanoplasmonic structures for sensing, wave guide, and microscopy, of using DNA origami conjugate with nanoparticles [10] have been proposed [3], [8].

Along with this direction, programmed (which means DNA origami was priori designed to connect together) onedimensional assembly of DNA origami without [13] and with [14] conjugating nanomaterial in the buffer solution was demonstrated. However, research efforts of DNA nanotechnology are not restricted in these directions but expanding on other directions, such as 2-D algorithmic assembly [15] and DNA nanomechanical devices with actuator function [16].

However, attempts to self-organize the DNA origami on a patterned substrate has not been succeeded yet. Several related works have been reported, such as selective deposition of DNA origami on the patterned gold [17] and silicon dioxide or diamond-like carbon [18] and DNA origami trapping between two electrode patterned on a silicon dioxide using dielectrophoretic force [19], [20]. All these efforts are not enough to realize selforganized DNA origami pattern on a substrate by arranging multiple DNA origami with different functionality selectively at a specific position in a specific sequence and interact with functional element of MEMS on the substrate. This is an essential demand to realize a nanosystem.

\section{DIRECTED SELF-ORGANIZATION OF A NANOSYSTEM}

A methodology recently proposed by the author who has been using DNA as an intelligent adhesive of functional microcomponents [21]-[23] makes it possible to realize a system in which functional nanocomponents are arranged at a specific position in a specific sequence according to the program. Sequence of nanocomponent arrangement is externally controlled, and the binding between nanocomponents is done by self-assembly. So, this methodology is called as a directed selforganization of nanosystem, and its concept is schematically shown in Figure 2.

The DNA origami with 100-nm scale is used as a functional building block on which nanomaterials, such as nanoparticles and nanotubes, are arranged precisely using addressing information of DNA origami scaffold. To assemble the DNA origami, ssDNA is used as an intelligent adhesive. Different ssDNAs, which act as coded adhesive to bond adjacent DNA origami building block, are laid out at their four sides. The assembly between DNA origamis is carried out using linker ssDNAs, and the assembly sequence is controlled by applying corresponding linker ssDNAs sequentially according to a target layout of the DNA origamis on MEMS. Figure 2(a) shows self-organized structure that uses four different DNA origamis as building blocks, which have nanoparticle patterns with different configuration such as straight, tee, cross, and corner. These nanoparticle patterns can be applicable to various applications, such as nanoplasmonics, by arranging these DNA origamis at a specific 


\section{Interaction and cooperation of multiple nanocomponents with different features make it possible to generate a unique and useful functionality of a nanosystem.}

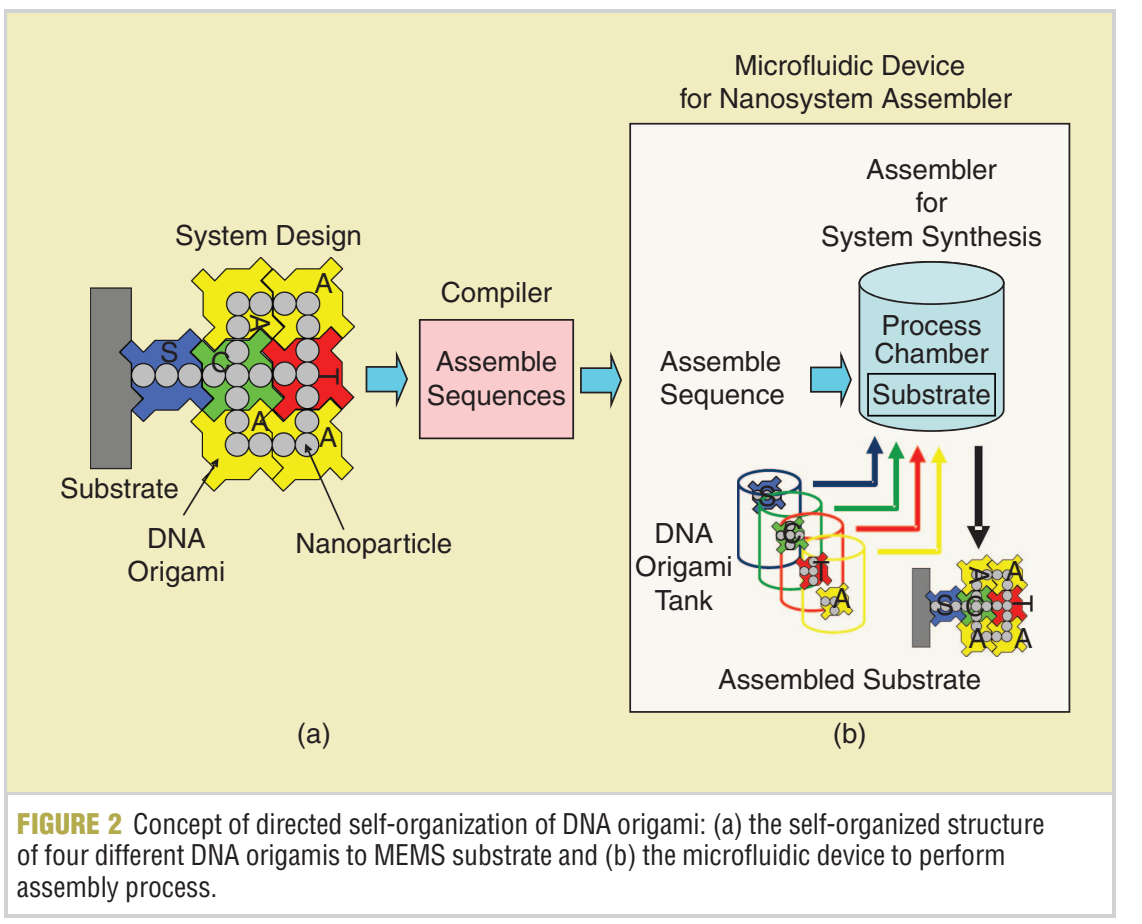

sequence based on a desired pattern on MEMS.

As describe earlier, the programmability can be realized using linker ssDNA between adjacent DNA origamis. Each side of DNA origamis, hereafter $\mathrm{B}_{i j}$ where $i(1$ to $n)$ represents a specific DNA origami type and $j$ (1 to 4 ) represents the specific side of a DNA origami, is modified by different $s \mathrm{DNA}_{\mathrm{B} i j}$. An assembly site $S$ on a substrate is also modified by ssDNAs. Now, let us assume $B_{11}$, side 1 of DNA origami $B_{1}$, should be assembled to a substrate site. A linker ssDNA that is composed of two types of ssDNA- $\mathrm{DNA}_{\mathrm{B} i} j^{\prime}$ as complementary ssDNA of $\mathrm{ssDNA}_{\mathrm{B} i j}$ and $\mathrm{ssDNA}_{\mathrm{s}}$ ' as complementary ssDNA of $\mathrm{ssDNA}_{s}$ - is prepared. The number of linker ssDNA required to realize directed arrangement of $n$ kinds of DNA origami building blocks is $2 n(4 n+1)$. This number can be further minimized by taking into account the symmetry of a building block, e.g., from 136 to 28 in the case of example shown in Figure 2. As shown in Figure 2, assemble sequence is generated based on the provided target design and transferred to a nanosystem assembler. This nanosystem assembler performs the assembly process repeatedly based on the generated sequence. The nanosystem assembler is realized as a microfluidic device, which is composed of an assembly chamber, several reservoirs containing DNA origamis and linker ssDNAs, and fluidic control components, such as valves, pumps, mixer, and separator. A MEMS substrate is set to this assembly chamber, and its temperature is controlled to perform self-organization process efficiently on the substrate. After one step of assembly process is finished, the excessive amounts of DNA origamis and linker ssDNAs are separated and recovered to the corresponding tanks and reused.

Preliminary experiment is ongoing. As a first step, three types of DNA origami (type E, F, and G), which was preprogrammed to be assembled in series in the order of E-F-G [13], were used to confirm the feasibility of using a microfluidic device. The top and crosssectional views of a microfluidic device are shown in Figure 3. The microfluidic device has six reservoirs that are connected to an assembly chamber located in the center. The pneumatically driven micropumps are arranged between the reservoirs and the assembly chamber to supply and drain the DNA origami and linker ssDNA dispersed solution. The additional micropump is arranged around the assembly chamber to generate perturbation of the fluidic flow inside the assembly chamber to improve the assembly yield and shorter the assembly process time. A mica substrate is placed in the center of the assembly chamber. Through the observation of the assembled DNA origami on the 
mica substrate by atomic force microscopy (AFM), it is confirmed that the appropriate perturbation increase the assembly yield of DNA origami. Once the optimization of perturbation and temperature condition is performed, as a next step, directed self-organization using non-preprogrammed DNA origamis and linker ssDNAs will be carried out. Then, as a final step, directed selforganization of DNA origami on a specific position of a MEMS substrate in a specific sequence will be challenged.

\section{REMAINING ISSUES AND PROSPECTS}

Research on directed self-organization of DNA origamis on MEMS to realize a nanosystem was introduced as a part of research on SENS. The following are the several remaining issues to be addressed intensively. 1) How to achieve higher assembly performance? Balance of long-range force determined by perturbation during assembly process and short-range force determined by DNA origami design and substrate modification at a certain temperature will be the key. 2) How to align the first DNA origami with the electrodes and/ or the waveguides on MEMS? The most straightforward approaches are functionalizing the circuit ends or fabricate template pattern for DNA origami at the adjacent position of the circuit ends using ebeam lithography. 3) How to transfer energy from the electrodes and/or waveguides on MEMS to a selforganized DNA origami structure and vice versa? Although the required energy transfer efficiency depends on the application, higher is better especially for a sensing application. Thus, an interface circuit/structure design is crucial. 4) How to stabilize the selforganized DNA origami structure at dry and relatively high temperature (at least $150{ }^{\circ} \mathrm{C}$ ) environment eventually in which the nanosystem is operated? There are two possible approaches: one is stabilizing DNA origami by incorporating additional chemicals into DNA origami structure, and the other is to take off (decompose)
DNA origami at the last step and leave the self-organized structure of nanomaterials on MEMS.

A number of articles on DNA nanotechnology are published every month, and the effort to solve these issues are increasing, so the author believe DNA nanotechnology will surely become a bridge between a nanomaterial and a nanosystem.

\section{ACKNOWLEDGMENTS}

I thank all my collaborators and colleagues, especially Prof. H. Sugiyama, Prof. M. Endo, Prof. Gwobin Lee, and Prof. J.G. Korvink. This work was supported by KAKENHI (22310081).

\section{ABOUT THE AUTHOR}

Osamu Tabata (tabata@me.kyoto-u. ac.jp) received his M.S. and Ph.D. degrees from Nagoya Institute of Technology, Japan, in 1981 and 1993, respectively. $\mathrm{He}$ is a professor at the Department of Micro Engineering, Kyoto University, Japan, and is currently engaged in the research of micro/nano process, MEMS, and micro/nano system synthetic engineering. $\mathrm{He}$ is a senior research fellow at Freiburg Institute for Advanced Studies and has visiting Professorship for Senior International Scientists at the Chinese Academy of Science.

\section{REFERENCES}

[1] O. Tabata, "Micro/nano assembly as a key to SENS (synthetic engineering for nano systems)," Electrochemical Soc. Trans, vol. 16, no. 15 , pp. 49-64, 2008

[2] N. C. Seeman, "Nucleic acid junctions and lattice," J. Theor. Biol, vol. 99, pp. 237-247, 1982.

[3] N. C. Seeman, "Structural DNA nanotechnology: Growing along with nano letters," Nano Lett, vol. 10, pp. 1971-1978, 2010.

[4] P. W. K. Rothermund, "Folding DNA to create nanoscale shapes and patterns," Nature, vol. 446, no. 16, pp. 297-302, Mar. 2006.

[5] C. J. Morris, S. A. Stauth, and B. A. Parvis, "Self assembly for microscale and nanoscale packaging: Steps toward self-packaging," IEEE Trans. Adv. Packag, vol. 28 , no. 4, pp. 600 611,2005

[6] J. Fang and K. F. B öhringer, "Self-assembly, comprehensive microsystems," J. Micromech. Microeng., vol. 1, pp. 403-427, 2007.

[7] D. Halley and D. A. Winkler, "Towards consistent concepts of self-organization and self assembly,' Complexity, vol. 14, no. 2, pp. 10-17, 2008.

[8] A. Kazuya and M. Komiyama, "DNA origami: Fold, stick, and beyond," Nanoscale, vol. 2, pp. 310-322, 2010.
[9] Y. Ke, S. M. Douglas, M. Liu, J. Sharma, A. Cheng, A. Leung, Y. Liu, W. M. Shih, and H. Yao, "Multilayer DNA origami packed on a square lattice," J. Amer. Chem. Soc, vol. 131, pp. 15903-15908, 2009

[10] B. Ding, Z. Deng, H. Yao, S. Cabrini, R. N. Zuckermann, and J. Bokor, "Gold nanoparticle self-similar chain structure organization by DNA origami," J. Amer. Chem. Soc, vol. 132, pp. 3248-3249, 2010.

[11] H. T. Maune, S. P. Han, R. D. Barish, M. Bockrath, W. A. Goddard III, P. W. K. Rothemund, and E. Winfree, "Self-assembly of carbon nanotubes into two-dimensional geometries using DNA origami templates," Nat. Nanotechnol, vol. 5, pp. 61-66, 2010.

[12] A. Kuzyk, K. T. Laitinen, and P. Torma, "DNA origami as a nanoscale template for protein assembly," Nanotechnology, vol. 20, pp. 235305-235309, 2009.

[13] M. Endo, T. Sugita, Y. Katsuda, K. Hidaka, and H. Sugiyama, "Programmed-assembly system using DNA jigsaw pieces," Chem. Eur. J, vol. 16, pp. 5362-5368, 2010.

[14] N. Stephanopoulos, M. Liu, G. J. Tong, Z. Li, Y. Liu, H. Yan, and M. B. Francis, "Immobilization and one-dimensional arrangement of virus capsids with nanoscale precision using DNA origami," Nano Lett, vol. 10, pp. 2714-2720, 2010.

[15] K. Fujibayashi, R. Hariadi, S. H. Park, E. Winfree, and S. Murata, "Toward reliable algorithmic self-assembly of DNA tiles: A fixedwidth cellular automaton pattern," Nano Lett, vol. 8, pp. 1791-1797, 2008.

[16] H. Gu, J. Chao, S. J. Xiao, and N. C. Seeman, "A proximity-based programmable DNA nanoscale assembly line," Nature, vol. 465 , no. 13 , pp. 202-205, 2010.

[17] A. E. Gerdon, S. S. Oh, K. Hsieh, Y. Ke, H. Yan, and H. T. Soh, "Controlled delivery of DNA origami on patterned surfaces," Small, vol. 5, no. 17, pp. 1942-1946, 2009.

[18] R. J. Kershner, L. D. Bozano, C. M Micheel, A. M. Hung, A. R. Fornof, J. N. Cha, C. T. Rettner, M. Bersani, J. Frommer, P. W. K. Rothemund, and G. M. Wallraff, "Placement and orientation of individual DNA shapes on lithographically patterned surfaces," Nat. Nanotechnol, vol. 4, pp. 557-561, 2009.

[19] A. Kuzyk, B. Yurke, J. J. Toppari, V. Linko, and P. Torma, "Dielectrophoretic trapping of DNA origami," Small, vol. 4, no. 4, pp. 447-450, 2008.

[20] V. Linko, S.-T. Paasonen, A. Kuzyk, P. Torma, and J. J. Toppari, "Characterization of the conductance mechanisms of DNA origami by AC impedance spectroscopy," Small, vol. 5 , no. 21, pp. 2382-2386, 2009.

[21] T. Kusakabe, T. Tanemura, Y. Higuchi, K. Sugano, T. Tsuchiya, and O. Tabata, "DNA mediated sequential self-assembly of nano/ micro components," in Proc. 21st IEEE Int. Conf. Micro Electro Mechanical Systems, Tucson, $A Z$, Jan. 13-17, 2008, pp. 1052-1055.

[22] T. Tanemura, G. Lopez, R. Sato, K. Sugano, T. Tsuchiya, O. Tabata, M. Fujita, and M. Maeda, "Sequential and selective self-assembly of micro components by DNA grafted polymer," in Proc. 22nd IEEE Int. Conf. Micro Electro Mechanical Systems, Sorrento, Italy, Jan. 25-29, 2009, pp. 184-187.

[23] G. Lopez, T. Tanemura, R. Sato, T. Saeki, Y. Hirai, K. Sugano, T. Tsuchiya, O. Tabata, M. Fujita, and M. Maeda, "DNA-grafted-polymer mediated self-assembly of micro components," in Proc. 5th IEEE Int. Conf. Nano/Micro Engineered and Molecular Systems, Xiamen, China, Jan. 20-23, 2010, pp. 248-252. 